\title{
On the Design of Coded Modulation for Fiber Optical Communications
}

\author{
Alex Alvarado ${ }^{\S}$, G. Liga ${ }^{\dagger}$, Tobias Fehenberger*, and Laurent Schmalen ${ }^{\ddagger}$ \\ ${ }^{\S}$ Signal Processing Systems (SPS) Group, Eindhoven University of Technology (TU/e), Eindhoven, The Netherlands \\ Optical Networks Group $(O N G)$, University College London (UCL), London, UK \\ ${ }^{*}$ Institute for Communications Engineering, Technical University of Munich, Munich, Germany \\ $\stackrel{\ddagger}{\ddagger}$ Nokia Bell Labs, Stuttgart, Germany \\ alex.alvarado@ieee.org
}

\begin{abstract}
In this paper we review achievable information rates (AIRs) for coded modulation. Using simple examples, AIRs are shown to be versatile design tools for fiber optical communication systems.
\end{abstract}

OCIS codes: (060.4080) Modulation, (060.4510) Optical communications.

\section{Coded Modulation}

The optical fiber channel is ultimately band-limited. Because of this, the need of designing bandwidthefficient transceivers is an active area of research. The most natural way of achieving this is via multi-level modulation combined with soft-decision (SD) forward error correction (SD-FEC), a combination known as coded modulation (CM). Different coded modulation flavors exist, the most popular ones being trellis coded modulation [1], bit-interleaved coded modulation [2,3], and multilevel codes [4, 5]. $\mathrm{CM}$ has become a key technology for fiber optical communications, as shown in Fig. 1. This figure shows the number of occurrences of "MQAM" in the OFC proceedings in the last 15 years. Although the number of occurrences is a very rough metric (and can certainly be improved), this figure does show a clear trend: QPSK and 16QAM are here to stay, and 64QAM and 256QAM are slowly becoming more important.

Ungerboeck's celebrated trellis-coded modulation [1] was very popular because the receiver could find the most likely coded sequence using a single, low-complexity decoder, that exploited the nonbinary (NB) trellis structure of the code. With the advent of powerful binary SD-FEC such

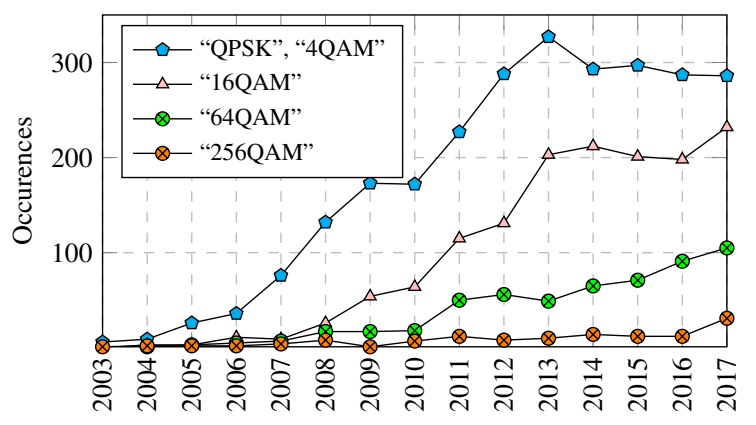

Fig. 1. Number of "MQAM" in OFC proceedings. low-density parity-check (LDPC) codes, however, modern fiber optical CM transceivers use a receiver based on a single binary FEC.

Until a few years ago, pre-FEC bit-error rate (BER), symbol error rate (SER), Q-factor, and error vector magnitude (EVM) were the standard performance metrics in the optical communications community. Recently, however, this paradigm has been changing and achievable information rates (AIRs) are being used more. In this paper, we discuss the use of AIRs as a tool to design CM transceivers with both NB and binary FEC. By means of simple examples, AIRs are shown to be very powerful design metrics.

\section{Achievable Information Rates: Three Applications}

AIRs indicate the number of information bits per symbol that can be reliably transmitted through the channel and are at the core of Shannon's celebrated concept of channel capacity [6]. Because of its definition, one of the key advantages of using AIRs as performance metrics is that they are inherently related to FEC. Unlike uncoded metrics like the pre-FEC BER, SER, EVM, or Q-factor, AIRs give an indication of the amount of information bits that can be reliably transferred through a channel. While uncoded metrics are related to bits before and after the demapper, AIRs deal with the information bits before and after FEC. AIRs allow fair comparisons of different constellations, DSP, decoding and 
nonlinearity compensation techniques, and can also be used for post-FEC error prediction. An AIR for CM based on NB codes is the mutual information (MI) [8-10]. For binary FEC, however, the relevant quantity is the GMI [3, 7, 11]. In this paper we consider equally likely symbols only, however, the extension of GMI to nonuniform symbols can be done, as shown in $[12$, Sec. VI]. In what follows, we show three applications of AIRs.

\subsection{Application 1: Throughput Prediction}

Fig. 2 (left) shows the MI (solid lines) for three different 8QAM constellations $\left(\mathscr{C}_{1}, \mathscr{C}_{2}\right.$ and $\left.\mathscr{C}_{3}\right)$ as well as the capacity of the 4D AWGN channel. These curves can be used to show the potential rate gains offered by the constellation $\mathscr{C}_{3}$. Fig. 2 (left) also shows the required $E_{b} / N_{0}$ for NB-LDPC codes with 5 different code rates to achieve a post-FEC error probability below $10^{-4}$ (more details in [10]). These results show that the MI can be used to predict the performance of NB-LDPC codes and NB modulations, where the gap between markers and lines is due to the suboptimality of the codes. Fig. 2 (right) shows results for binary LDPC codes with 7 different rates and two different modulation formats. In this figure, both MI (solid lines) and GMI (dashed lines) are shown, as well as the performance of the code (more details in [7]). From this figure, it is clear that binary LDPC codes follow the GMI prediction rather than the MI. This figure also shows that although $\mathscr{C}_{4,256}$ has higher MI compared to 16QAM, this gain does not translate into rate gains with LDPC codes because the GMI of $\mathscr{C}_{4,256}$ is lower than the one of 16QAM.
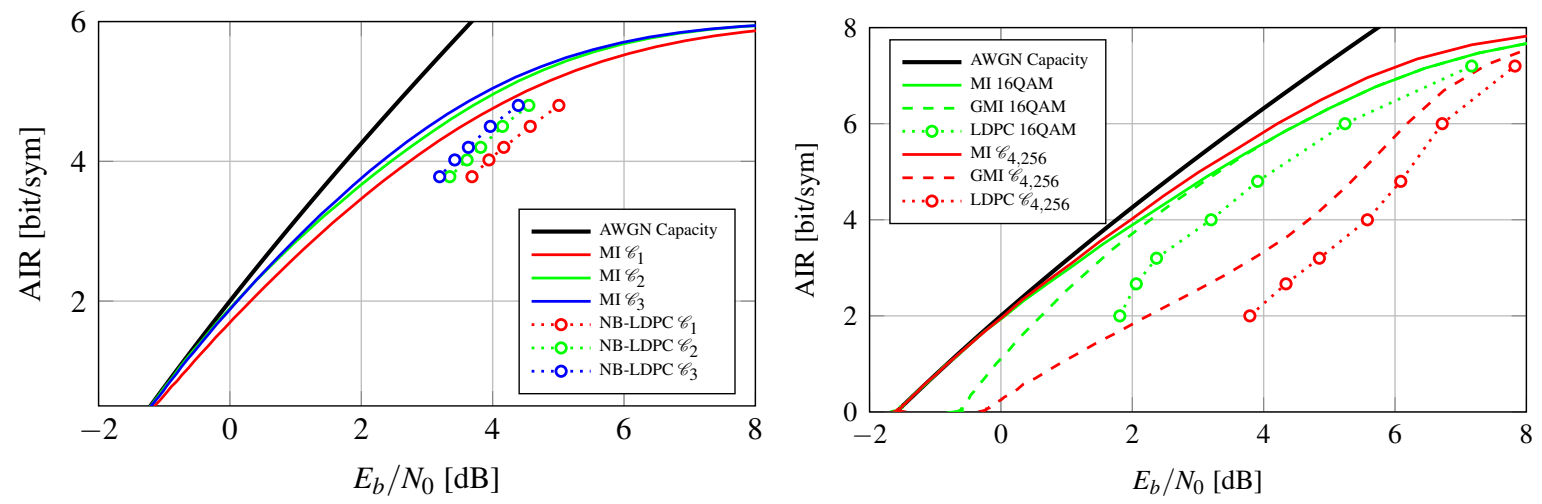

Fig. 2. CM with NB-LDPC codes [10] (left) and binary LDPC codes [7] (right).

\subsection{Application 2: Reach Increase}

Fig. 3 shows two examples of AIRs versus transmission distance and highlights the fact that AIRs can be used to predict the reach increase in optical systems. For example, Fig. 3 (left) shows different modulation formats and nonlinearity compensation techniques (EDC and DBP), which can be directly compared against each other (for the same target AIR). These results show that, for multi-span, multi-channel, optical transmission (single-channel) DBP with 64QAM offers a potential reach increase of app. $1,000 \mathrm{~km}$ at $8 \mathrm{bit} / \mathrm{sym}$ with respect to EDC. Fig. 3 (right) shows that for the system in [8], the gains offered by probabilistic shaping are equivalent to those offered by DBP. The use of AIRs can therefore be used to guide the CM design, and also to decide on how to trade complexity and performance.
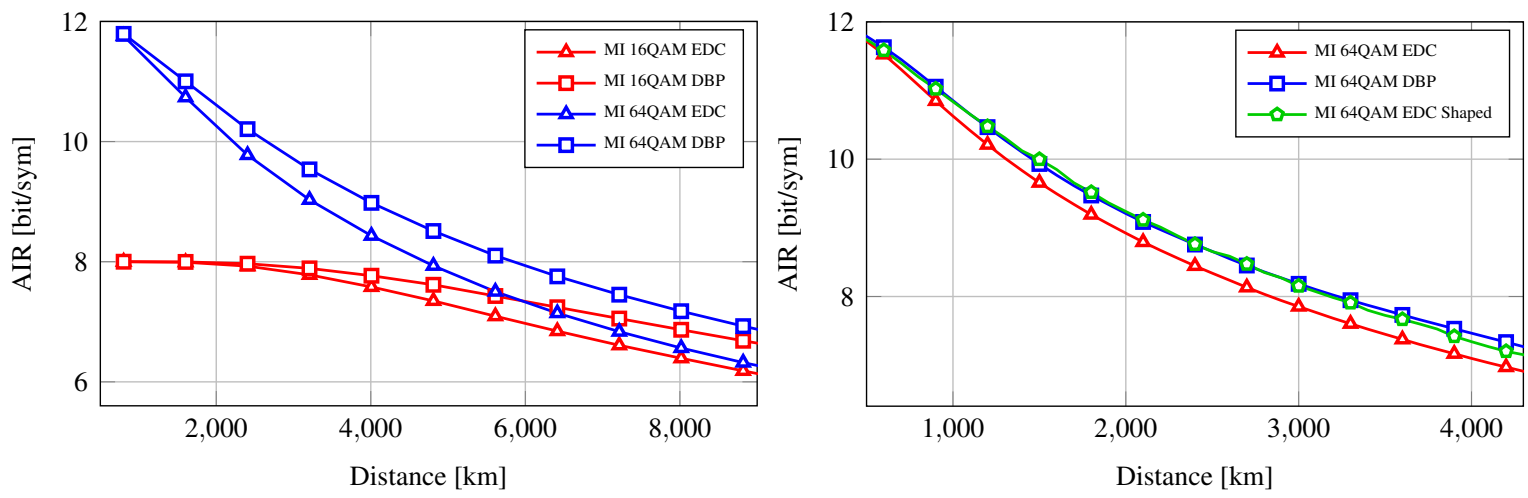

Fig. 3. AIRs versus transmission distance in [9] (left) and [8] (right). 


\subsection{Application 3: Post-FEC Performance Prediction}

AIRs can also be used as decoding thresholds. The error probability after FEC can be accurately predicted by considering the MI and GMI, for NB-SD-FEC and binary SD-FEC, respectively. Consider for example an AWGN channel, the NB-LDPC codes from [10], and three different 8QAM constellations from [10, Fig. 3]. The post-FEC SER results are shown in Fig. 4 (left), where different markers represent different modulation formats. This figure shows how the MI (normalized by the number of bit/symbol $\log _{2} M$ ) is a good predictor of the post-FEC SER. Similar results are shown in Fig. 4 (right) for binary LDPC codes from the DVB-S2 standard (randomly punctured to obtained the rate shown) and three different modulation formats (4QAM, 16QAM, and 64QAM). In this case the decoder is binary and the normalized GMI is the quantity that correctly predicts the post-FEC BER for all modulation formats (different markers). The main result in Fig. 4 is that normalized MI and GMI are very good decoding thresholds for SD-FEC.
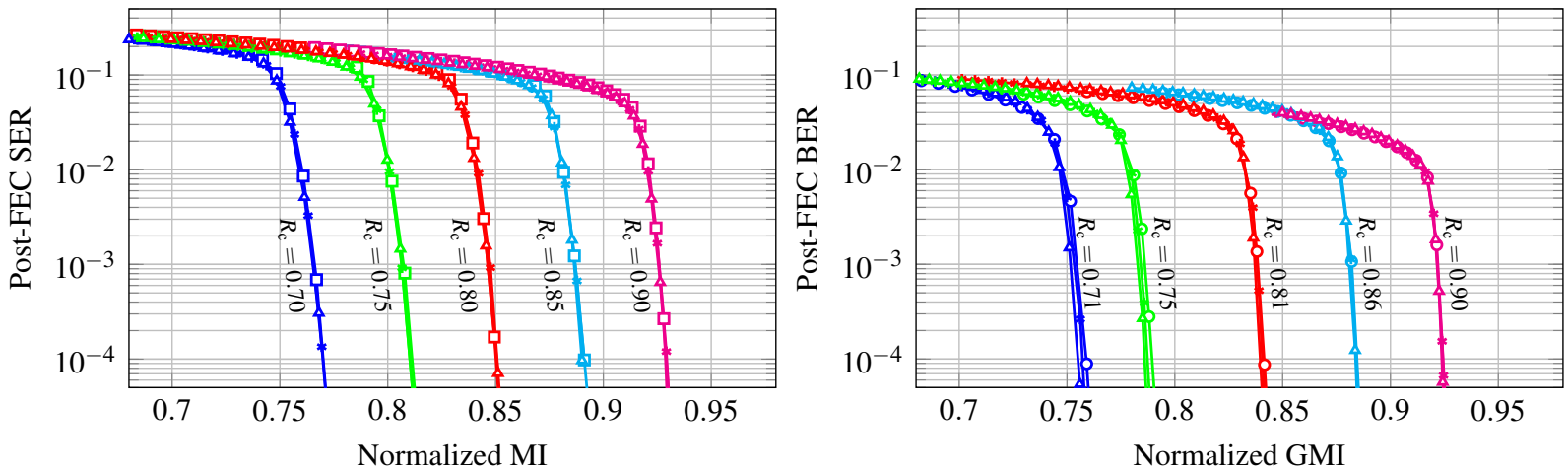

Fig. 4. Post-FEC prediction for NB in [10] (left) and binary LDPC codes (right).

\section{Conclusions}

The merits of achievable information rates for the design of coded modulation in fiber optical communications were discussed. These rates are in general more difficult to calculate than, e.g., pre-FEC BER. However, they are more versatile and represent a much more natural option for the design metric of modern (coded) fiber optical communication systems.

\section{References}

1. G. Ungerboeck, "Channel coding with multilevel/phase signals," IEEE Trans. Inf. Theory, vol. 28, no. 1, pp. 55-67, Jan. 1982.

2. E. Zehavi, "8-PSK trellis codes for a Rayleigh channel," IEEE Trans. Commun., vol. 40, no. 3, pp. 873-884, May 1992.

3. L. Szczecinski and A. Alvarado, Bit-Interleaved Coded Modulation: Fundamentals, Analysis and Design, John Wiley \& Sons, 2015.

4. H. Imai and S. Hirakawa, "A new multilevel coding method using error-correcting codes," IEEE Trans. Inf. Theory, vol. IT-23, no. 3, pp. 371-377, May 1977.

5. U. Wachsmann, R. F. H. Fischer, and J. B. Huber, "Multilevel codes: Theoretical concepts and practical design rules," IEEE Trans. Inf. Theory, vol. 45, no. 5, pp. 1361-1391, July 1999.

6. C. E. Shannon, "A mathematical theory of communications," Bell System Tech. Journal, vol. 27, pp. 379-423/623-656, July/Oct. 1948.

7. A. Alvarado and E. Agrell, "Four-dimensional coded modulation with bit-wise decoders for future optical communications," J. Lightw. Technol., vol. 33, no. 10, pp. 1993-2003, May 2015.

8. T. Fehenberger, A. Alvarado, P. Bayvel, and N. Hanik, "On achievable rates for long-haul fiber-optic communications," Opt. Express, vol. 23, no. 7, pp. 9183-9191, Apr. 2015.

9. G. Liga, A. Alvarado, E. Agrell, and P. Bayvel, "Information rates of next-generation long-haul optical fiber systems using coded modulation," J. Lightw. Technol., vol. 35, no. 1, pp. 113-123, Jan. 2017.

10. L. Schmalen, A. Alvarado, and R. Rios-Müller, "Performance prediction of nonbinary forward error correction in optical transmission experiments," J. Lightw. Technol., vol. 35, no. 4, pp. 1015-1026, Feb. 2017.

11. A. Martinez, A. Guillén i Fàbregas, G. Caire, and F. Willems, "Bit-interleaved coded modulation revisited: A mismatched decoding perspective," in Proc. IEEE International Symposium on Information Theory (ISIT), Toronto, ON, Canada, July 2008.

12. G. Böcherer, F. Steiner, and P. Schulte, "Bandwidth efficient and rate-matched low-density parity-check coded modulation," IEEE Trans. Commun., vol. 63, no. 12, pp. 4651-4665, Dec. 2015. 